\title{
APPLICATION OF MODERN METHODS OF SOCIOMETRIC ANALYSIS FOR MODELLING PERSONNEL OPTIMAL BEHAVIOR AT THE ENTERPRISE
}

\begin{abstract}
The article focuses on the analysis of the sociomapping method used for modeling the personnel optimal behavior at the enterprise, as well as on the method efficiency evaluation within the framework of the conducted research. It is suggested to apply the sociomapping method for evaluating the efficiency of the enterprise corporate culture and for the analyzing the enterprise collective organizational behavior in general.

The key benefits and drawbacks of the sociomapping method application are considered within the framework of the enterprise social networks analysis.

Key words: personnel, HR management, personnel behavior management, sociometry, sociomapping, organizational behavior, corporate culture.
\end{abstract}

Formation of the problem. Forming the efficient personnel behavior management system requires an effective tool for modeling optimal processes in the microenvironment of personnel. The tool should cover all the socio-psychological factors, cultural and behavioral characteristics of communications. For this purpose, the method of sociomapping, based on the model of fuzzy sets has been suggested. The topicality of the research lies in the fact that the sociomapping method has never virtually been applied to the analysis of personnel social networks at domestic enterprises.

Analysis of recent research and publications. Foreign experience comprises a variety of sociometric analysis models. The method of sociomapping has been relatively recently elaborated as part of sociometry and social networks theory. R. Bahbouh and R. Warrenfeltz $[1 ; 2]$ were the first to suggest the method of sociomapping for social network analysis. All further research with the application of sociomapping method was concentrated on forming development programmes for personnel management, team efficiency analysis, interpersonal cooperation and team communication. However, particular significance of personnel behavior within the context of organizational culture was not taken into account in these works. Thus, the models proposed in the research works require further detailed review and specification. Moreover, analyzing the recent research which was conducted using instruments of sociomapping, we have found that none of them have been used for the modeling of optimal personnel behavior in a changing, dynamic environment.

Research objectives. The main objectives of the article are analyzing and researching the effectiveness of the sociomapping method, describing its key benefits for modeling personnel optimal behavior at the enterprise.

Presentation of the main research material. In the context of this research we address the personnel behavior management in view of cultural aspect influences, using the set of tools of corporate culture and socio-psychological motives of behavior.

Taking into consideration the determination of person's behavior by his or her psychological structure, it is technologically impossible to unify the process of personnel behavior management by shaping it into certain standard. It is to some extent a creative process that it requires creativity and knowledge of psychology by managers. It is therefore unreasonable to apply only the methods of mathematical and economic modeling, as we are faced with the socio-psychological characteristics which cannot be assigned any values or given quantitative evaluation.

Forming the efficient personnel behavior management system needs an effective tool for modeling optimal processes in the microenvironment of personnel covering all the sociopsychological factors, cultural and behavioral characteristics of communications. With this aim we suggest the method for modeling the effective behavior of personnel at the enterprise. This method, unlike others, reveals the process of forming the effective personnel behavior and 


\section{H. M. Zakharchyn, Y. M. Kosmyna}

covers all the socio-psychological aspects of formal, informal and cultural networks among the staff of the enterprise.

Sociomapping is a new method for conducting profound analysis of socio-economic systems which reveals hidden structural elements within the complex socio-psychological human resources system by analyzing its dynamics [4].

Sociomapping is also a unique visualization instrument, which enables comprehensive depiction of complex systems, such as work teams. It provides both experts and laymen with the opportunity for swift and intuitive orientation within the analyzed system and provides them with support for making decisions on its management [7].

Sociomapping monitors the most important characteristics of inter-elemental relationshipsfrom capturing the degree of stability and the composition of these relationships (including their inner conflicts and disagreements), to mapping communication currents (the degree of their functionality in each direction) and uncovering the weaknesses in the social system structure. Additionally, it reflects the system's dynamic development and tension build-up, and allows for the short-term prediction of future behavior (conflicts, performance issues etc.) [1].

It is common knowledge that numerous factors can promote the development and success of the enterprise. But none of them is as significant as the management ability to manage the complex human resources system, their behavior, in particular, accomplishing the goals of the organization efficient operation with a cohesive team. The effective personnel behavior management leads to the enterprise development and achievement of its goals and objectives.

The aim of the sociomapping method is to diagnose the causes of low personnel efficiency, reveal positive and negative impact of communication in professional, informal and personal networks of the personnel. The sociomapping method is based on fuzzy sets theory and mathematical topology. The theoretical principles of the latter have been actively developing and being applied to modeling of decision-making process under the conditions of incomplete data [5].

The sociomapping method combines the information on human resources system obtained from various sources. Such important interelement connections as stability level of personnel behavior and level of inner tensions can be controlled by means of sociomapping. It is also possible to plan the level of functional efficiency of each worker that is, in this or other way, an element of informal network of the enterprise staff. Sociomapping can be useful in revealing weaknesses of the socio-psychological system of an organization. The method permits the shortterm prediction of the personnel behavior (level of staff cohesiveness, level of personnel affiliation, conflicts, and effectiveness level).

Technological realization of the sociomapping method envisages preliminary surveys of the enterprise workers by means of individual interviewing. The aim of the method realization is to prepare the map of formal and informal connections within the personnel.

The respondents were asked specific questions in order to define the level of professional and non-professional contacts within the enterprise staff. They were also invited to estimate the level of affiliation using the scale of $1-10$ points, in accordance with the model of fuzzy sets.

The survey provided the evaluation of attribution of each separate factor to the corresponding set of fuzzy terms (linguistic variables) using the point scale (1-10; 1 - low level, 5 - average level, 10 - high level). The proposed model is based on the assumption that if the degree of affiliation exeeds 0.5 points, the efficiency grows. Proceeding from this assumption, relatively average degree of affiliation can be regarded as one of the predictors of efficiency.

Based on the information received from 91 surveyed workers of PLC Leoni Wiring UA $\mathrm{GmbH}$, the conceptual socio-maps of formal and informal networks have been produced. Figure 1 depicts the typical example of the formal network socio-map at PLC Leoni Wiring UA GmbH enterprise. Figure 2 depicts its informal network.

The sociometric map of personnel formal network at the estimated enterprise (fig. 1) traces effective and non-effective interpersonal connections [6] in accordance with the assumptions made where the lines represent formal connections between personnel of the network [3]. 


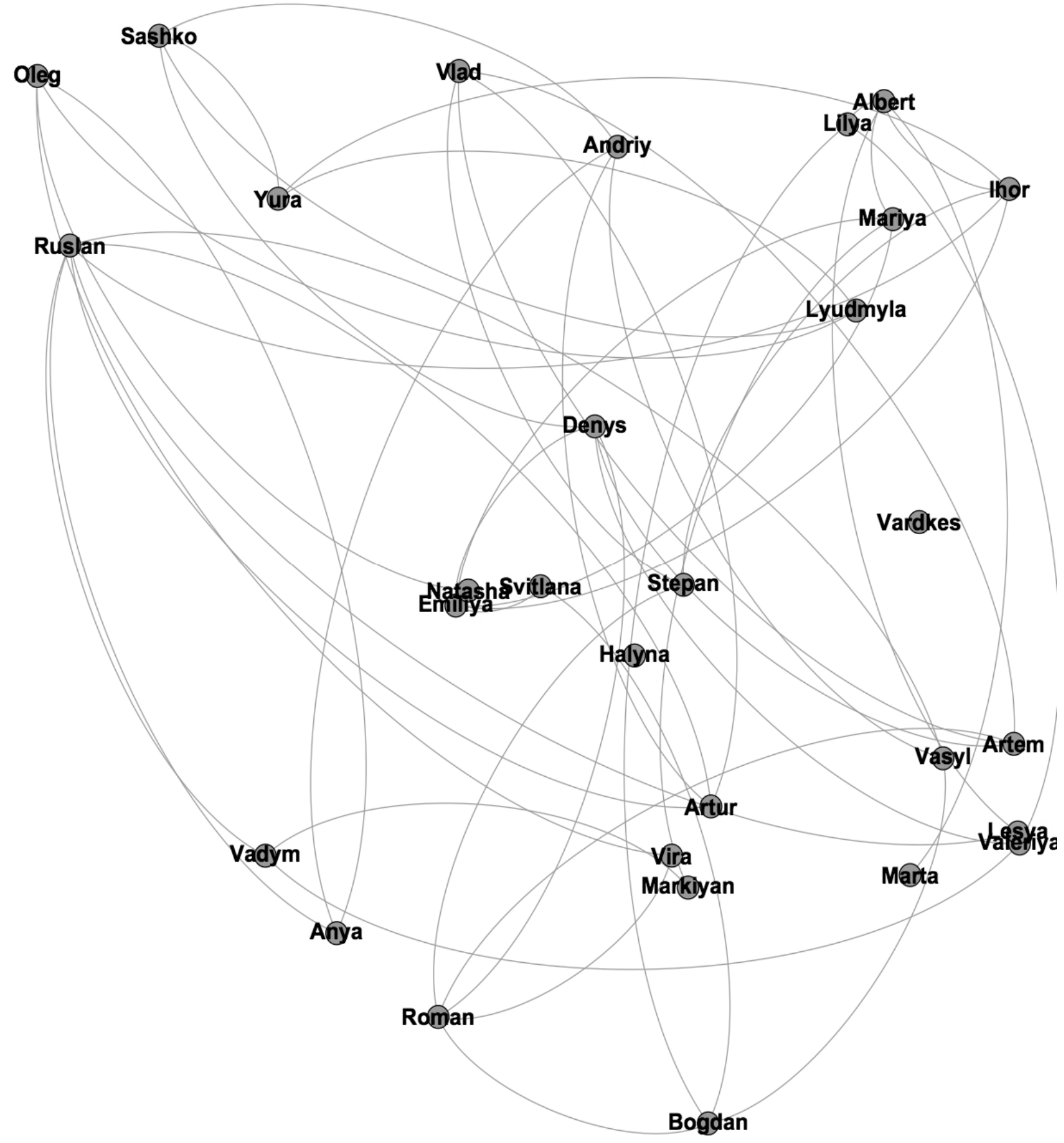

Fig. 1. Sociometric map of the personnel formal network at PLC Leoni Wiring UA GmbH.; done by author

After analyzing the informal network sociometric map of Leoni Wiring UA GmbH (fig. 2) it was established that the enterprise has three informal groups of personnel interpersonal connections which proved the inefficiency of corporate culture implementation. Separate groups of this subculture perform different tasks and functions moving the enterprise away from common goals and declared values. Thus, the key tasks of the enterprise manage- ment in such conditions are to form and adapt corporate culture by means of cross-cultural communication instruments, evaluate the efficiency level of both groups in order to determine priority targets in personnel behavior management in the long run.

The lines in figure 2 reflect the closeness of connections inside the informal network; the length of the line between vertices corresponds to the of connections between them. 


\section{H. M. Zakharchyn, Y. M. Kosmyna}

In graph theory closeness is a centrality measure of a vertex within a graph (network). Vertices that are 'shallow' to other vertices have higher closeness. Closeness is preferred in network analysis to mean shortest-path length, as it gives higher values to more central vertices, and so is usually positively associated with other measures such as degree. In the network theory, closeness is a sophisticated measure of centrality. It is defined as the mean geodesic distance (i.e., the shortest path) between a vertex and all other vertices reachable from it [7].
Three interpersonal close groups of workers (also called 'subcultures') are showed on the map for the illustration purposes. Each subject depicted on the map has the membership degree, clustering coefficient and the distance-between-nodes (connections) defined. Comparative analysis of formal and informal networks of the researched enterprise was conducted covering these average indexes as well as the closeness centrality index and diameter of the network (graph).

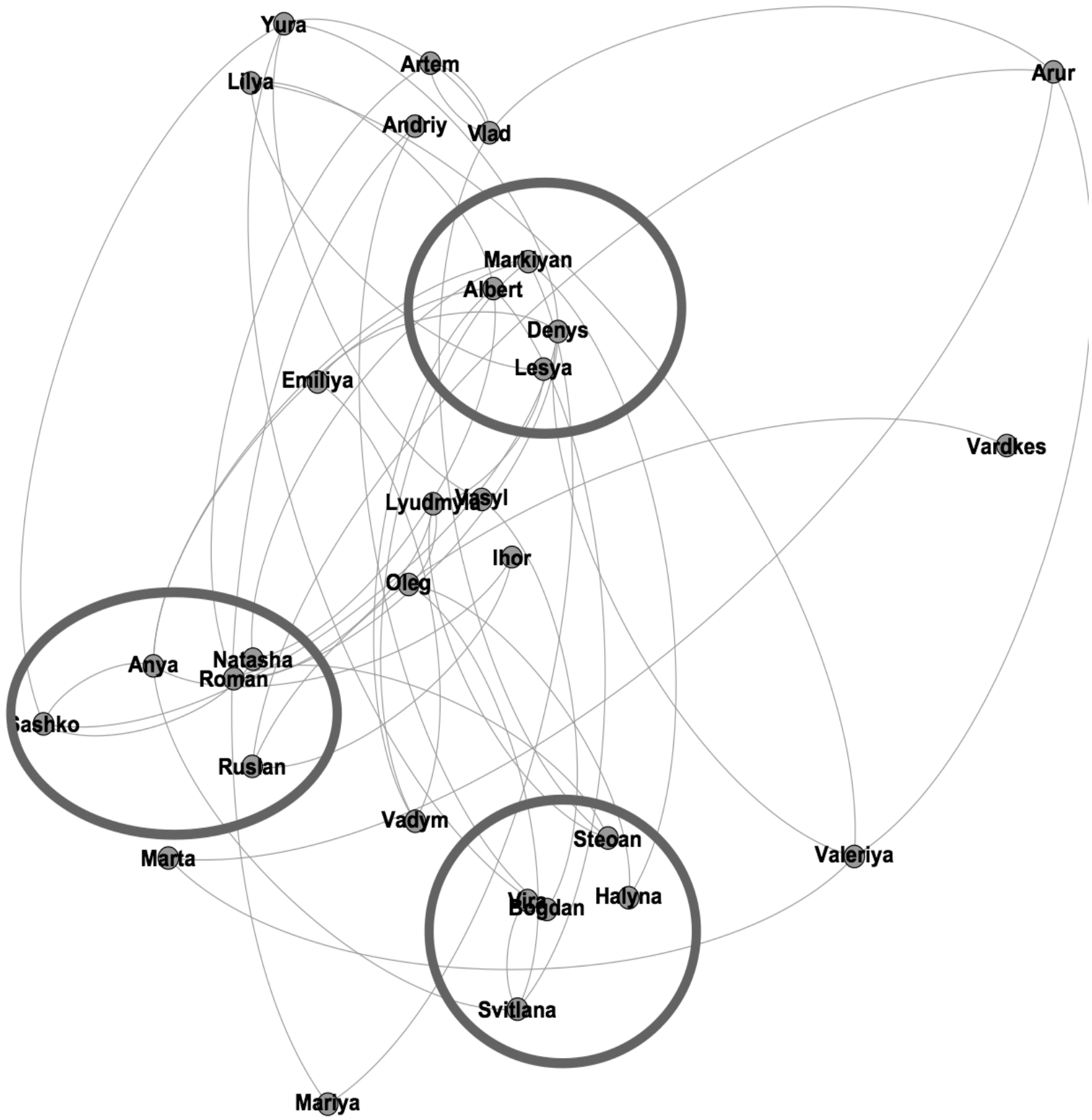

Fig. 2. Sociometric map of informal network at PLC Leoni Wiring UA GmbH.; done by author 
The average membership degree shows the level of staff cohesiveness. As the average level of affiliation grows, the effectiveness of team work improves as well. Average clustering coefficient reveals closeness of connections within a team. It serves to validate the hypothesis about the existence of close subcultures in the team.

Betweenness is a centrality measure of a vertex within a graph. Vertices that occur on many shortest paths between other vertices have higher betweenness than those that do not. [6] In other words, the betweeness centrality index reveals the distance between all the pairs of vertices within the network. Lower index stands for better level of team communication which facilitates efficiency of personnel behavior management. Higher clustering coefficient in the informal network means the increase of its efficiency. Forming small sub-cultures within formal networks in professional relations at the enterprise does not promote development and transparency. Calculated indexes at the researched enterprise speak for quite a high level of efficiency of the formal network.

Relatively low indexes of informal network are indicative of weak informal network at the enterprise which in its turn can hamper the formation process of optimal behavior of the personnel and the development of efficient corporate culture of the enterprise.

Table 1

Comparison of formal and informal networks at PLC Leoni Wiring UA GmbH

\begin{tabular}{|l|c|c|c|}
\hline \multicolumn{4}{|c|}{ FORMAL NETWORK } \\
\hline & $\begin{array}{c}\text { Average } \\
\text { membership } \\
\text { degree index }\end{array}$ & $\begin{array}{c}\text { Average } \\
\text { clustering } \\
\text { coefficient }\end{array}$ & $\begin{array}{c}\text { Average } \\
\text { betweeness } \\
\text { centrality index }\end{array}$ \\
\hline $\begin{array}{l}\text { Leoni } \\
\text { Wiring }\end{array}$ & 3.267 & 0.053 & 2.738 \\
\hline \multicolumn{4}{|c|}{ INFORMAL NETWORK } \\
\hline $\begin{array}{l}\text { Leoni } \\
\text { Wiring }\end{array}$ & $\begin{array}{c}\text { Average } \\
\text { membership } \\
\text { degree index }\end{array}$ & $\begin{array}{c}\text { Average } \\
\text { clustering } \\
\text { coefficient }\end{array}$ & $\begin{array}{c}\text { Average } \\
\text { betweeness } \\
\text { centrality index }\end{array}$ \\
\hline & 1.6 & 0.069 & 4.113 \\
\hline
\end{tabular}

Source: Author

The benefits of proposed method for modeling the optimal personnel behavior based on sociomapping are the following:
- relatively low labor content as regards construction and usage;

- objective possibility of receiving data for modeling by means of surveying;

- flexibility of the method as in conditions of change there is a possibility of quickly respond to changing mood and behavior of the personnel;

- the method enables quantitative evaluation of qualitative characteristics such as the team cohesiveness, loyalty, formal and informal connections etc.

- Enables holistic analysis of efficiency of personnel behavior management at the enterprise.

\section{Conclusions}

The method for visualization of team profiles has been suggested. The main advantage of the method proposed is that it is able to visualize the similarity of profiles in a way which is more friendly to human perception than when presenting profiles by line-graphs or just through a matrix of numbers. The final visualization through the sociomap picture provides a general overview of differences between profiles.

From the research done the following conclusions can be made: the method of sociomapping will facilitate prediction of the future personnel behavior; it will enable analysis of behavioral characteristics of each separate worker of the enterprise with the aim to maximally reveal the workers' potential and realize it; it will enable to analyze the process of forming sub-cultures within the enterprise for their constructive integration into the unified corporate culture; the sociomapping method allows to determine objectively the level of efficiency of corporate culture formation, reveal its strengths and weaknesses; it will enable to increase personnel efficiency using the informal network advantages.

We are convinced that Sociomapping is a promising method for analysis and visualization of the social ties development andd also for the detection of potentially critical situations.

\section{References}

1. Bahbouh R. Sociomapping. Dissertation thesis / R. Bahbouh // Charles University in Prague. 2004. 


\section{H. M. Zakharchyn, Y. M. Kosmyna}

2. Bahbouh R. Sociomapping of teams / R. Bahbouh. Praha: Dar Ibn Rushd \& QED GROUP. - 2011.

3. Carrington P. J. Models and Methods in Social Network Analysis (Structural Analysis in the Social Sciences) / P. J. Carrington, J. Scott, S. Wasserman // Cambridge University Press, 344 p. -2005.

4. Höschl C. Visualization and Testing Team Profiles / C. Höschl // Degree thesis, Faculty of Mathematics and Physics, Charles University in Prague. - 2010.
5. Klebanova T. S. Modeli otsinky efektyvnosti sanacii pidpryemstv na osnovi nechitkyh mnozhyn / T. S. Klebanova, O. V. Panasenko // Aktualni problem ekonomiky. - 2007. - \#7. P. 158-165

6. Scott J. Social Network Analysis / J. Scott. - NY: SAGE Publications Ltd; Third Edition edition. 2012. $-216 \mathrm{p}$.

7. TEAM SOCIOMAPPING. A Method of Increasing the Effectiveness of Teams and Team Coaching. QED Group. - 2009. 